\title{
Limits in the validity of Arrhenius predictions for field ageing of a silica filled polychloroprene in a marine environment
}

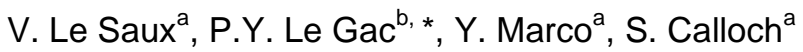 \\ a Laboratoire Brestois de Mécanique et des Systèmes (EA 4325), ENSTA Bretagne/UBO/ENIB, 2 rue F. Verny \\ 29806 Brest Cedex 9, France \\ b IFREMER, Marine Structures, centre de Brest, BP 70, 29280 Plouzané, France \\ *: Corresponding author : P.Y. Le Gac
}

\begin{abstract}
:
Accelerated ageing in natural sea water at different temperatures from $20^{\circ} \mathrm{C}$ to $80^{\circ} \mathrm{C}$ was performed on a silica filled polychloroprene rubber. Degradation when monitored with mechanical properties at both macroscopic and microscopic scale led to a large increase of the modulus coupled with a strong decrease of strain and stress at break. Data from tensile tests were used for lifetime prediction with an Arrhenius extrapolation for modulus, strain and stress at break. The validity of this lifetime prediction was evaluated using a 23 year-old sample aged in natural conditions. Strain at break could be predicted using an Arrhenius extrapolation with an activation energy of $50 \mathrm{~kJ} / \mathrm{mol}$. However, an extrapolation based on linear Arrhenius behavior did not apply for modulus and stress at break due to the presence of a degradation profile across the sample. These observations confirmed that strain at break is not governed by bulk properties of samples but by the degradation rate at the external surface of the sample (i.e. in contact with water).
\end{abstract}

Keywords : polychloroprene rubber ; accelerated and natural ageing tests ; Arrhenius extrapolation

\section{Introduction}

A clear understanding of ageing processes is crucial to guarantee the long term durability of a new product or to reduce the frequency and thus the cost of maintenance operations. This is a challenging task because ageing is a 
complex phenomenon that covers a broad spectrum of multidisciplinary domains including the definition of accelerated ageing tests, the identification of the ageing mechanisms, the evaluation of the consequences on the mechanical properties and finally the simulation of real components that integrate the gradients induced by ageing [1]. In order to keep reasonable test and product development durations, accelerated ageing is mandatory. The most common approach to accelerate ageing uses elevated temperature tests combined with an Arrhenius type extrapolation to predict the value of a given parameter for lower temperatures and longer times (see for example ISO standard test method 11346 for rubbers [2]). The Arrhenius extrapolation is widely used for lifetime prediction of polymers in different environments [3, 4, 5]. This common application is due to the fact that this approach is easy to perform and can be, in some cases, relevant. Nevertheless, Arrhenius extrapolation in the field of polymer degradation exhibits some limitations and many authors reports that temperature effects on degradation kinetics can not always be described using the Arrhenius equation. [6, 7, 4]. Several factors are involved but two major reasons can be emphasized. First, when the chemical reactions involved in polymer degradation vary between high temperature and low temperature, it is not possible to extrapolate degradation kinetics using a simple time/temperature superposition [7, 4]. However, this can be overcome by using a mechanistic approach of the degradation [8, 9], which relies on a description of each chemical steps involved in the overall degradation. Second, when degradation involves the diffusion of a reactive component from the external environment to the bulk of the material, non uniform ageing in the polymer thickness with depth can occur. This phenomenon is due to a competition between diffusion and reactive consumption; when diffusion is faster than reaction, then degradation is uniform in the sample thickness whereas when reaction is faster than diffusion then the resulting degradation is non uniform [10, 11, 12, 13, 14]. When degradation is not uniform with depth, an extrapolation of macroscopic properties using the simple Arrhenius time/temperature equation will obviously not be relevant ; in this case only edge information can be relevant.

Dealing with the marine ageing of rubbers, many studies have been published [15, 16, 17, 18, 19], but very few articles investigate the consequences of a marine environment on polychloroprene rubber (CR) [20] and none consider possible degradation profiles. This conclusion results from the good resistance of rubbers to this kind of environement [21, 15]. The results by Ab-Malek and Stevenson [16], Pegram and Andrady [17], Oldfield and Symes 
[18] or more recently Davies and Evrard [5] show that after very long periods of exposure, the behaviour of the studied materials remains almost identical to that of the unaged one. For all these reasons, there is a lack of experimental data allowing the establishment of Arrhenius extrapolation for marine aged rubbers [3, 5] and very few naturally aged rubber parts to check the relevance of these extrapolations have been examined [16]. Moreover, when naturally aged samples are available, results are rarely compared to accelerated ageing results.

The aim of this paper is to evaluate the influence of marine ageing on the mechanical behaviour of a polychloroprene rubber, both at macroscopic and microscopic scales, and to compare the predicted lifetime (using a linear Arrhenius behavior) to data from a naturally aged sample (a 23 year old offshore floating export line). First, we present the experimental details, and describe the material used here and the accelerated ageing protocols that are necessary to evaluate the ageing consequences at laboratory time scale. Experimental devices used here to evaluate the evolution of the mechanical behaviour are also presented. Second, we present the experimental results. Finally, a comparison between the results obtained from the accelerated ageing tests and the naturally aged ones is presented.

\section{Experimental details}

\subsection{Material}

This study is focused on a filled polychloroprene rubber used as a coating of offshore floating flow lines. The composition of the material is given in Table 1 and the main mechanical properties are given in Table 2, It is worth noting that there is no silane coupling agent in the formulation. In order to perform accelerated ageing, square plates of $2 \mathrm{~mm}$ thickness and $250 \mathrm{~mm}$ width were manufactured by an industrial partner from the same material batch, in order to ensure the reliability of mixing and moulding conditions.

\subsection{Natural ageing}

The naturally aged sample originated from the coating layer of an offshore floating export line. This flow line was used in the Atlantic Ocean (near the Cameroun coast) for 23 years at $10 \mathrm{~m}$ depth. Seawater temperature varies from $15^{\circ} \mathrm{C}$ to $25^{\circ} \mathrm{C}$ in this area during the year and $\mathrm{pH}$ is around 8.2. The flowline was still meeting the structural mechanical requirements after its 


\begin{tabular}{cc}
\hline components & content (phr) \\
\hline Chloroprene Rubber & 100 \\
ZnO & 6 \\
$\mathrm{MgO}$ & 4 \\
plasticizer & $<10$ \\
carbon black & 6 \\
silica & 46 \\
stearic acid & 2 \\
antioxidants & 2 \\
accelerators & 1 \\
\hline
\end{tabular}

Table 1: Formulation of the studied material.

\begin{tabular}{cc}
\hline properties & CR \\
\hline density & 1.4 \\
hardness (Shore A) & 68 \\
microhardness $[\mathrm{MPa}]$ & 3.2 \\
$\pi_{\text {rupt }}[\mathrm{MPa}]$ & 17.4 \\
$\varepsilon_{\text {rupt }}[\%]$ & 670 \\
\hline
\end{tabular}

Table 2: Main mechanical properties of the studied material before ageing. 
withdrawal from service. This naturally aged sample is $2.3 \mathrm{~mm}$ thick and meant to protect the structure from any environmental degradation, and did not reveal any visible damage after its service life. The compound used for the accelerated ageing tests has exactly the same formulation as the one used in the flow line.

\subsection{Accelerated ageing}

In order to be as close as possible to the service conditions, specimens were immersed in several tanks filled with natural seawater coming directly from the Brest estuary and maintained at different temperatures for different durations (cf. Table 31). Water was continually renewed using a peristatic pump leading to the replacement of the vessel volume $(60 \mathrm{~L})$ every $24 \mathrm{~h}$ without any modification of the temperature. $2 \mathrm{~mm}$ thick plates were removed periodically from the tanks.

\begin{tabular}{cccc}
\hline $20^{\circ} \mathrm{C}$ & $40^{\circ} \mathrm{C}$ & $60^{\circ} \mathrm{C}$ & $80^{\circ} \mathrm{C}$ \\
\hline- & - & 16 & 16 \\
- & 30 & - & 30 \\
50 & 50 & 50 & 50 \\
- & - & - & 79 \\
172 & 172 & 172 & 172 \\
- & 381 & 381 & - \\
526 & 526 & - & - \\
686 & - & 686 & - \\
\hline
\end{tabular}

Table 3: Exposure times [days] for thermally accelerated aging under water immersion.

During the accelerated ageing, the samples will absorb some water according to diffusion mechanisms [21], leading to an increase in mass and volume. Even if no dramatic effects on the mechanical behaviour are expected [21], a reversible effect is generally noted in rubber modulus [22]. Unfortunately, the dependence of the mechanical behaviour on both the ageing degradation and the amount of water absorbed by the material does not allow for an objective comparison between various ageing conditions easy. To be able to compare the various ageing conditions, we chose to focus on dried samples. To be as unintrusive as possible, the samples were dried at $40^{\circ} \mathrm{C}$ in an inert atmosphere (N2 gas) until a constant weight was reached. This drying 
protocol was optimized and validated by a specific study detailed elsewhere [23].

\subsection{Tensile tests}

After the ageing and drying steps, the samples were cut from the sheets with a punch die in order to obtain normalized dumbbell shapes (type 2 from the standard ISO 37:2005). The choice to age sheets rather than dumbbell samples originates from the difference in the diffusion processes. Dealing with samples cut from sheets, the gradients will be 1D with only variations along the thickness and not along the width (as it would have been if dumbbell samples were aged), which is clearly easier to manage. The tensile tests were performed on a Lloyd LR5K + testing machine, equipped with a $1 \mathrm{kN}$ load cell. The tests were displacement controlled with a grip speed of $10 \mathrm{~mm} / \mathrm{min}$. A laser extensometer LASERSCAN200 was used to measure the true strain during testing. For each ageing condition, at least three samples were tested and the results averaged. In this paper strain and stress are related to nominal values, i.e. both values are calculated based on initial parameters (lentgh and section). In this paper three values will be considered : strain at break, stress at break and M100 which is modulus at 100\% i.e. the stress at this elongation.

\subsection{Hardness profiling}

\section{Sample preparation}

The elastic modulus profiles were obtained using a CSM-instruments micro-hardness tester with a Vickers tip. The samples cut from $2 \mathrm{~mm}$ thick sheets were embedded at room temperature in epoxy resin and subsequently polished with grinding media of decreasing granularity (down to grit size 800). The disc rotation speed was low in order to prevent sample heating. After grinding, a delay of $2 \mathrm{~h}$ was systematically applied in order to let the samples cool down to ambient temperature.

\section{Hardness tests}

A classical load controlled protocol was used, with a low preload in order to detect the surface contact (set to $10 \mathrm{mN}$ ), then a load controlled loading step (up to $100 \mathrm{mN}$ at an loading rate of $200 \mathrm{mN} / \mathrm{min}$ ), followed by a creep period of $30 \mathrm{~s}$ and then a load controlled unloading step (down to $0 \mathrm{mN}$ at a unloading rate of $200 \mathrm{mN} / \mathrm{min}$ ). A micro hardness (related to Young's 
modulus) was evaluated from linear section of the load-displacement curve using the following equation:

$$
H_{I T}=\frac{F_{\max }}{A_{p}}
$$

where $A_{p}$ is the projected area of the contact surface evaluated from the Oliver and Pharr methodology [24] and obviously related to measured penetration depth of tip in sample. Each point is an average of 5 measurements.

In the following, we will focus only on the normalized micro hardness, i.e. the value of the hardness divided by its initial value obtained on the unaged material. This choice is motivated by the fact that this micro-hardness quantity is strongly dependent on the affected volume and thus the microstructure of the material. Therefore, this value cannot be easily correlated to more classical hardness values such as macroscopic Shore and DIDC, but the variation of hardness can be easily interpreted.

\section{Results}

\subsection{Naturally aged sample}

Tensile curves obtained for an unaged and for the naturally aged sample are plotted in Figure 1. Natural ageing led to a significant change in mechanical behavior of the rubber. In fact, a large increase of the initial stiffness was observed, for example if we consider a specific strain of 100 the stress is around $1 \mathrm{MPa}$ for the unaged sample and $6 \mathrm{MPa}$ for the 23 year old sample. At the same time, a large decrease of ultimate properties was observed after natural ageing: in fact, strain at break decreased from 670 to $190 \%$.

In order to reveal (or not) the degradation heterogeneities, some modulus profiles [25, 23] were obtained for a naturally aged sample. The results are given in Figure 2. It should be mentioned that only the external side (on

the left) was exposed to seawater, which explains the asymmetrical profile obtained. The naturally aged sample exhibited a clear gradient between the skin and the core with a significant increase of the hardness on the side exposed to the seawater, whereas the right side (not exposed to the seawater) remained nearly equivalent to the unaged material. The slight increase of the hardness (compared to the unaged material) in the internal could be explained by the evolution of the material after its removal from service, rather than an influence of the marine ageing or by some slight changes in 


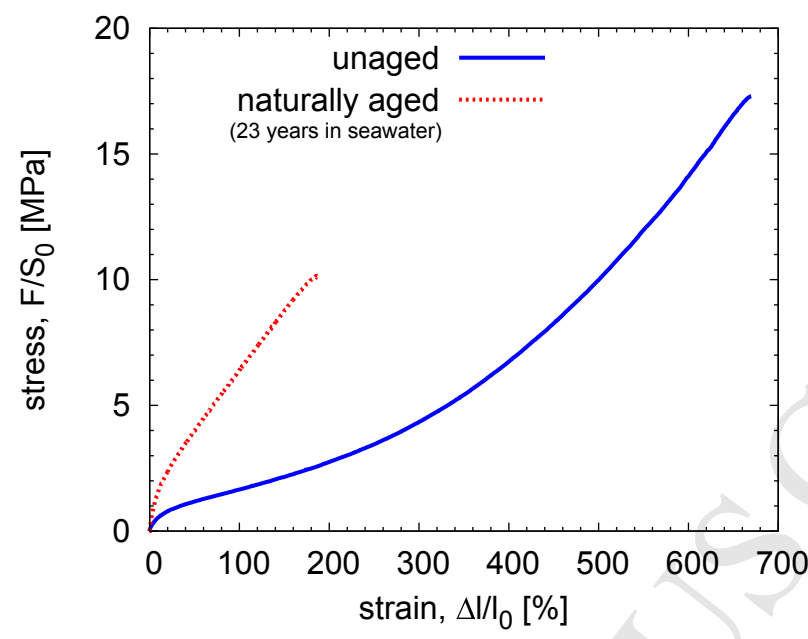

Figure 1: Tensile behaviour of the naturally aged sample and comparison to the unaged material showing a substantial loss in maximum load performance.

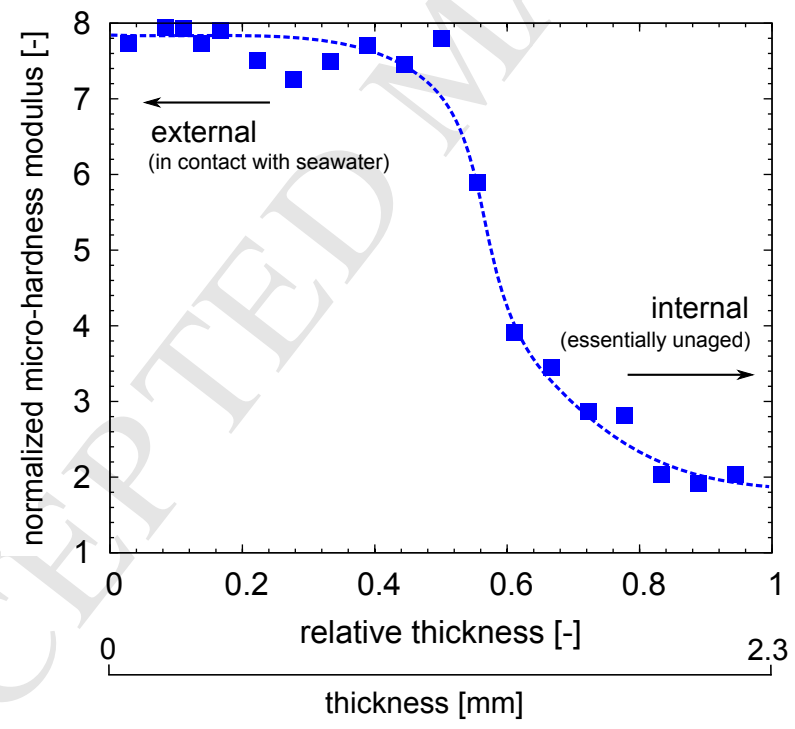

Figure 2: Evolution of the hardness across the thickness of the naturally aged sample.

the chemical structure of some of the ingredients.

Characterization of a 23 year old sample clearly showed a large change in 
mechanical behavior due to ageing in sea water. In fact, a large increase in rubber stiffness and loss of ultimate properties was observed. At the same time, ageing in service leads to a degradation profile across the sample thickness due to a limitation of the degradation depth by water diffusion. An ongoing question in the polymer ageing field is 'How to predict such evolution in a relative short time using laboratory tests?'. This will be discussed in the context of results obtained during accelerated ageing tests performed in sea water at different temperatures.

\subsection{Accelerated ageing between 20 and $80^{\circ} \mathrm{C}$}

Tensile Tests

Tensile behavior of $\mathrm{CR}$ after accelerated ageing at $80^{\circ} \mathrm{C}$ in sea water for different durations are plotted in Figure 3. Similarly to the naturally aged sample, ageing led to a significant increase of the initial stiffness (up to 200 $\mathrm{MPa}$ after 6 months) and a considerable drop of the ultimate properties (strain and stress at break). It was also observed that the longer the ageing duration, the more significant the changes of the tensile properties. These

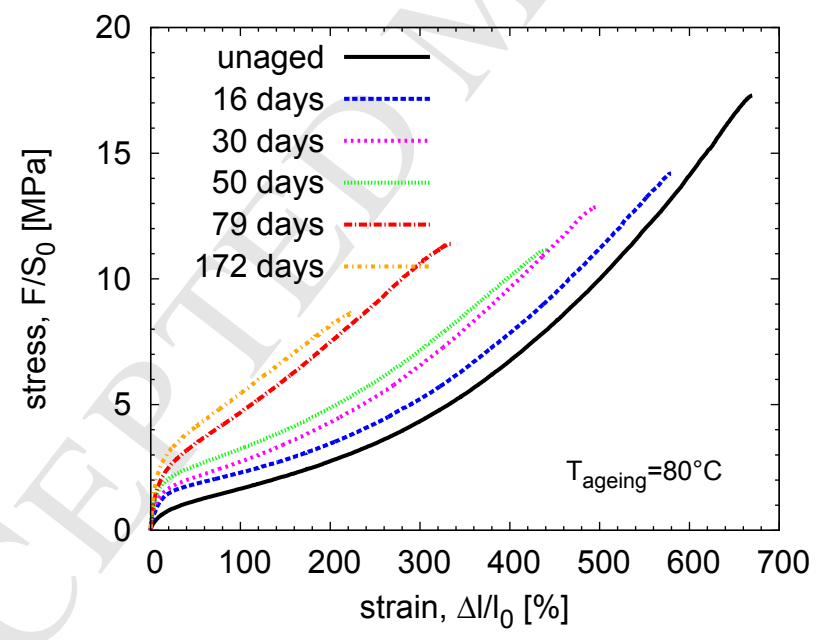

Figure 3: Stress/Strain curves obtained for ageing under water immersion at $80^{\circ} \mathrm{C}$.

results were observed for all ageing temperatures and a strong dependence of the degradation kinetics on the temperature is noted as illustrated in Figures 4, 5] and 6, which present the evolution of the normalized strain, stress at break and normalized modulus at $100 \%$ for the ageing conditions. Moreover, 
it should be emphasized that the tensile property observations are indeed very similar to the ones observed on the naturally aged sample, which highlights the relevance of the accelerated degradation pathway. This result is moreover supported by the complete physical analysis performed by Le Gac et al. [26].
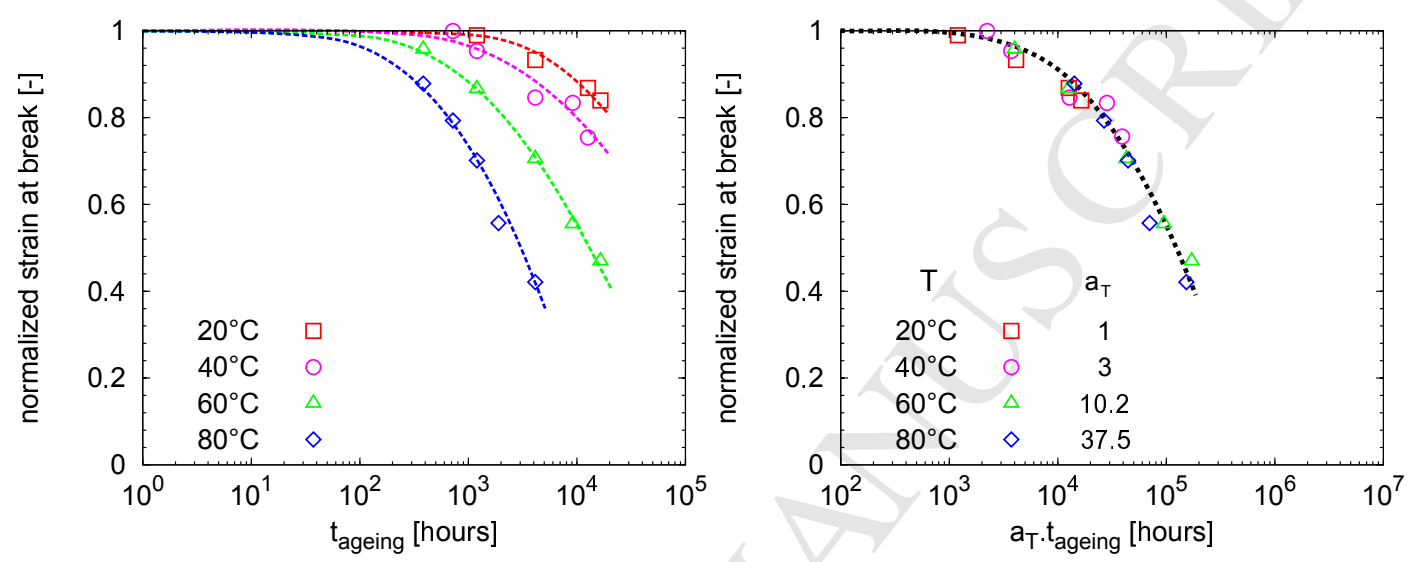

Figure 4: Evolution of the normalized strain at break (strain at break divided by the strain at break of the unaged material) as a function of ageing time for all the temperatures (a) and master curve (b).
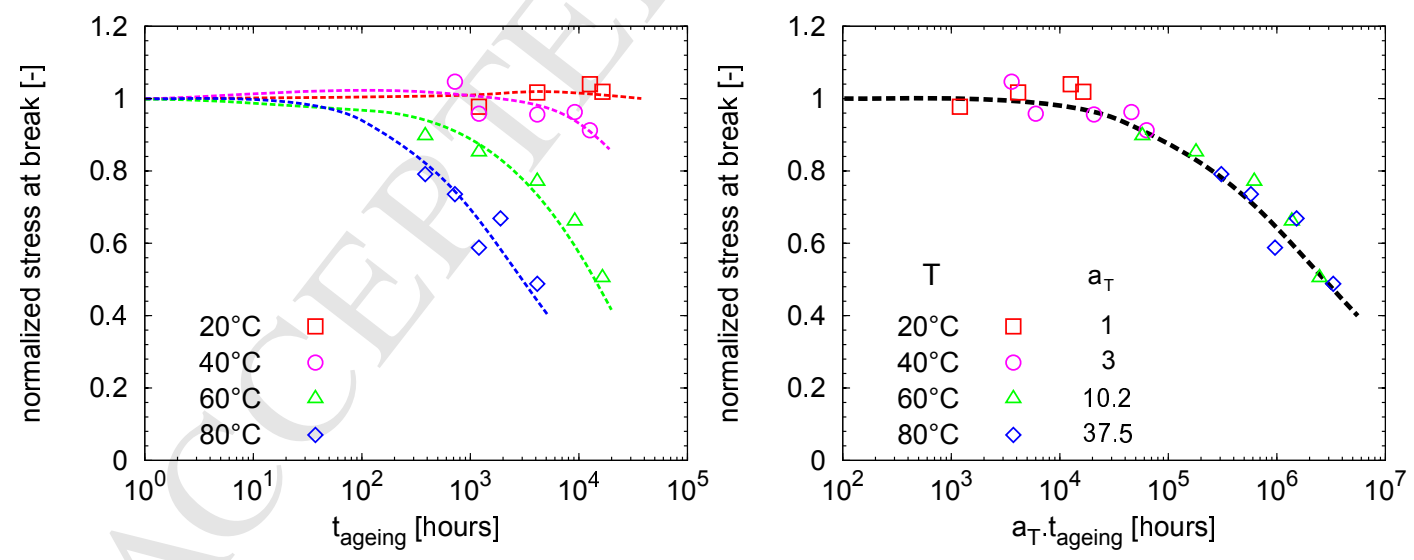

Figure 5: Evolution of the normalized stress at break (stress at break divided by the stress at break of the unaged material) as a function of ageing time for all the temperatures. 

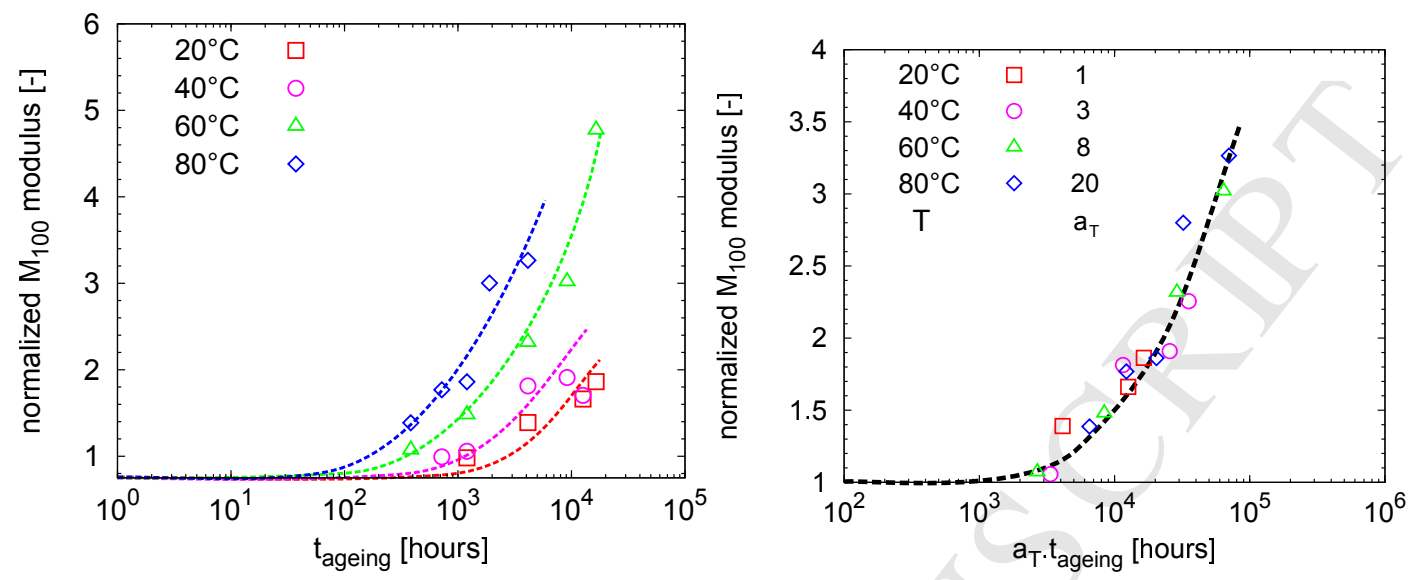

Figure 6: Evolution of the normalized modulus at $100 \%$ as a function of ageing time for all the temperatures (a) and master curve (b).

Using accelerated ageing in the laboratory, it is thus possible to produce similar changes in tensile behavior as for natural ageing in a reduced time frame. Based on these results, Time/Temperature superposition was performed according to the so called Arrhenius approach. This approach is based on the assumption that the degradation involves one unique chemical process activated by the temperature and whose rate $q$ is proportional to:

$$
q \propto \exp \left(-E_{a} / R T\right)
$$

In this equation, $E_{a}$ is the Arrhenius activation energy, $R$ is the gas constant and $T$ is the absolute temperature. The Arrhenius approach predicts that the curves obtained at the different accelerated ageing temperatures all have the same "shape" when plotted as a function of the logarithm of time. It is therefore possible to superpose all the data in order to get a master curve by defining shift factors $a_{T}$ :

$$
a_{T}=\exp \left[\frac{E_{a}}{R}\left(\frac{1}{T_{\text {ref }}}-\frac{1}{T}\right)\right]
$$

The reference temperature was chosen to be the lowest test temperature, i.e. $20^{\circ} \mathrm{C}$ in our case. Master curves for strain at breack, stress at break and modulus at $100 \%$ are respectively plotted in Figures 4 , 5 and 6 .

In order to assess degradation profile across sample thickness during accelerated ageing tests, local hardness measurements were performed. 


\section{Hardness profiling}

Hardness profiles as function of ageing time (Figure 7 - a contant temperature of $80^{\circ} \mathrm{C}$ ) and temperature (Figure 7 -b constant time of 172 days) are plotted in Figure 7. A clear gradient between the skin and the core of the sample was observed. The ratio between the skin and the core values increased as the severity of the ageing increased, i.e. higher temperatures and/or durations. The presence of gradients and their strong dependence on the ageing temperatures and durations implies that degradation is limited by water diffusion. This behavior is consistent with the general trend observed in well known Diffusion Limited Oxidation (DLO).
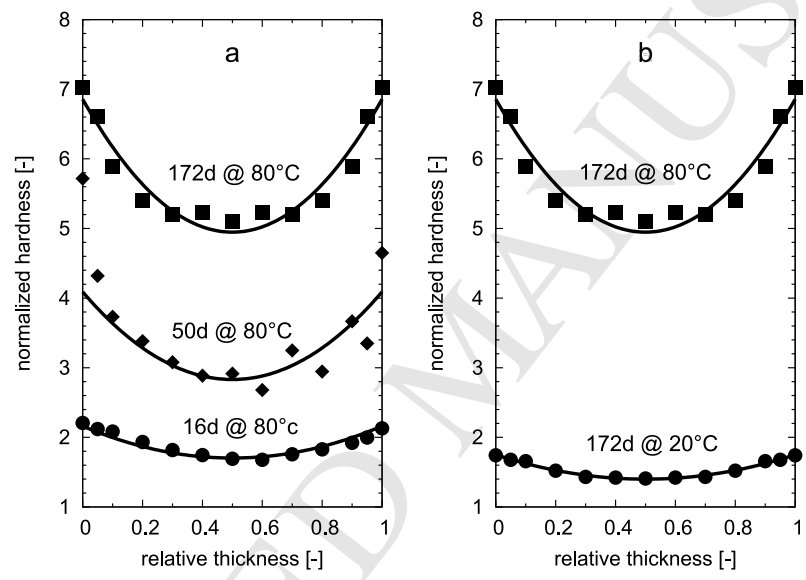

Figure 7: Micro-hardness profiling for various ageing durations for a given temperature (a) or various ageing temperature for a given duration (b).

Major degradation of a polychloroprene rubber was observed when used in sea water. Both natural and accelerated ageing led to a large increase of the polymer stiffness and decrease of ultimate tensile properties. Moreover, due to water diffusion limited degradation, hardness profiles across sample thickness were observed during ageing. This will be discussed next in the comparison between predicted behavior based on accelerated ageing results using a linear Arrhenius extrapolation and the actual measured properties on the 23 year old sample.

\section{Discussion and lifetime prediction}

Results presented in the previous section highlighted interesting features. Degradation consequences of silica filled polychloroprene in sea water can be 
summarized, from a mechanical point of view, as a large increase in the modulus of the material and an important decrease in ultimate tensile properties (stress and strain at break).

These trends were observed in both accelerated and natural ageing. Therefore we will focus on the possibility of using a linear Arrhenius behavior in order to make a life time prediction to compare with the actual ageing observed after 23 years in service.

\subsection{Validity of the Arrhenius prediction}

Historically, the so-called Arrhenius approach has been extensively used to analyze short-term high temperature accelerated ageing exposures and then to extrapolate these results to make long-term predictions at lower temperature, typically at the service temperature. The activation energy is determined using the previously defined shift factors (Figure 8). The straight line used for this identification is then extrapolated in order to get the shift factors related to the service temperature, based on the assumption that $E_{a}$ remains constant over the extrapolated temperature range. Using the

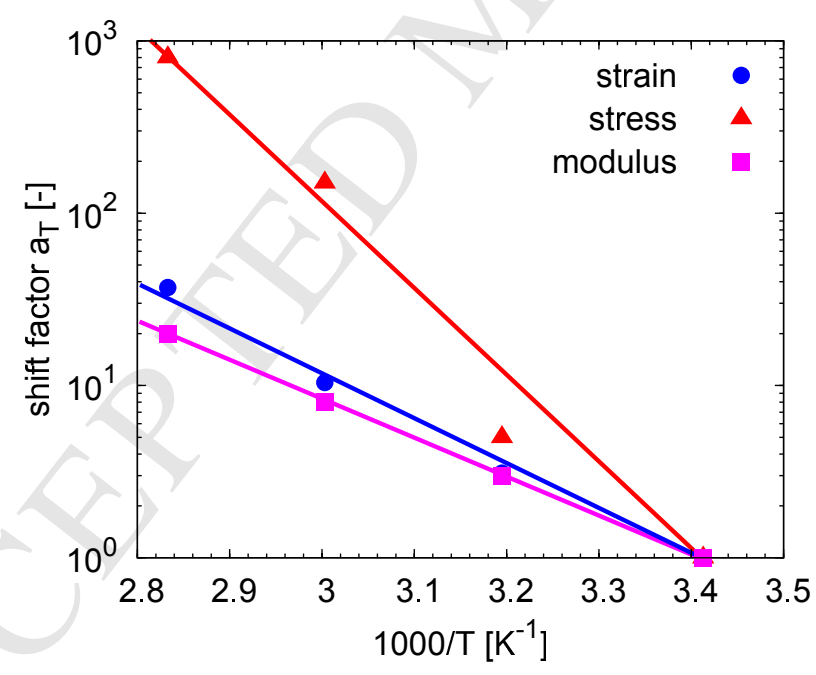

Figure 8: Identification of the activation energy for the strain at break indicator.

results obtained on the naturally aged sample, it is now possible to evaluate the applicability of the Arrhenius lifetime prediction. Figure 9 presents the master curve obtained from accelerated ageing in the laboratory and the 
point corresponding to the naturally aged sample.

For stress at break and modulus, predictions do not match the results

\begin{tabular}{cc}
\hline Properties & $E_{a}$ values $[\mathrm{kJ} / \mathrm{mol}]$ \\
\hline nominal strain at break & 52 \\
nominal stress at break & 100 \\
$M_{100}$ modulus & 42 \\
\hline
\end{tabular}

Table 4: Activation energies for the 3 mechanical indicators.

obtained on the 23 year old sample. This observation is explained by the presence of a gradient in the sample thickness revealed by micro hardness measurements (this point will be discussed in the next section).

Considering strain at break, Figure 9a clearly shows a good prediction using a linear Arrhenius relationship for this property. This behavior could be explained by the fact that elongation at break is governed by the weakest point in the sample [27], i.e. in this case the external surface of the sample which is directly in contact with water. This behavior has already been observed in the case of DLO [28] [29]. // However, it should be noted that the accuracy of the prediction depends largely on the temperature range used for accelerated ageing. In fact, in this case, because of the fast degradation kinetics, Arrhenius parameters were determined over a large temperature range (from 20 to $80^{\circ} \mathrm{C}$ ) and close to the service temperature.

Life time prediction using linear Arrhenius behavior is not valid for both stress at break and modulus. This is due to, at least, one phenomenon : the observed degradation profile across sample thickness, which is discussed below.

\subsection{Discussion of the degradation gradient in the field sample}

The field aging behavior exemplified by the material changes shown in Figure 8 is intriguing for a number of reasons. First of all such observations are rare: therefore, this is an excellent example of substantial aging effects occuring over long-term low temperature conditions. Second, there is sufficient chemistry to result in an aging process associated with significant hardness. Lastly, this process is clearly limited in depth and consistent with a diffusion limited degradation profile where aging has taken place over 23 years without saturation in physical property or homogenous degradation 


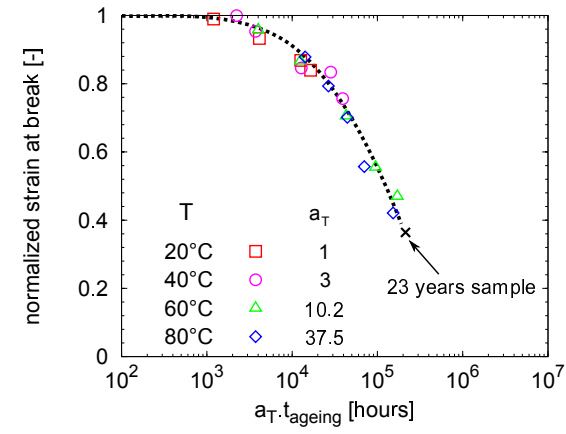

(a) strain at break master curve

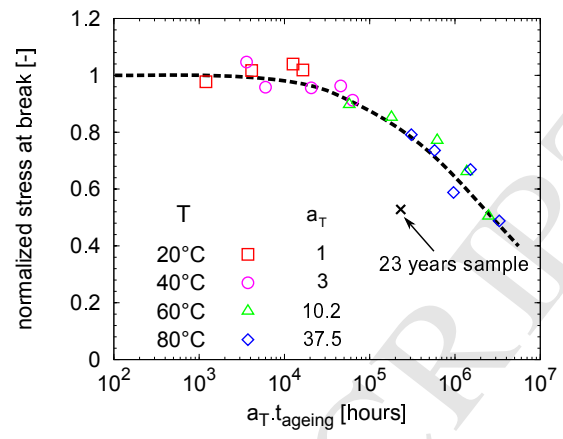

(b) stress at break master curve

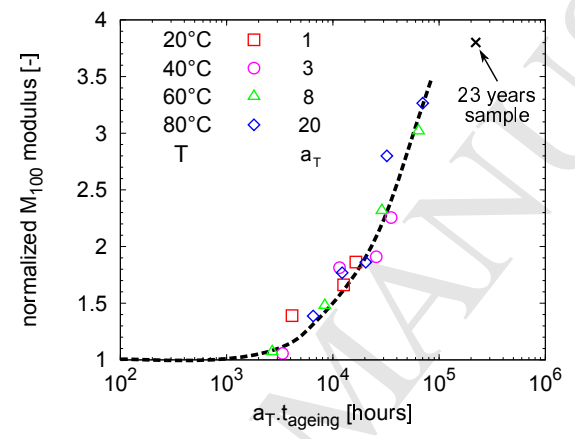

(c) M100 modulus master curve

Figure 9: Comparison between the master curves obtained for the (a) strain at break, (b) stress at break and (c) modulus and the data coming from the naturally aged sample (error bars are due to uncertainty in service temperature and the cross represents prediction for a mean value of $20^{\circ} \mathrm{C}$ )

across the thickness of this specimen. The question then arises as to how the underlying process resulting in this aging state should be discussed.

As shown elsewhere [26], for a number of reasons, namely oxidation is observed only in the first 200 microns and antioxidants remain present in the bulk even after 23 years, so oxidatively driven aging processes should be discounted for the degradation of this material. All evidence supports that hydrolytic degradation is involved in the aging of this filled neoprene material. Lacking any specific hydrolytic degradation rates and physical parameters like diffusion and permeation rates for this material, we can nevertheless discuss the features of the observed profile. It is quite possible that the profile may have developed as part of a diffusion limited profile that has shifted inwards with time resulting in the hardness plateau feature across the 
exterior half section of material. Similarly, it is also possible that the profile represents a slow aging behavior that occurred in an equilibrium condition over time. Lacking any more data, it is difficult to distinguish between these two aging processes, yet most importantly it is obvious that the feature of a diffusion limited degradation process is visible and hence a permeative ingress of a reagent must be in equilibrium with a consumption rate yielding the profile decay in the interior section. Can permeation be sufficiently slow or an aging rate sufficiently fast to yield this profile and at the same time result in a reasonable cumulative degradation state with time?

In terms of the physical description of diffusion limited degradation processes there is no difference between an oxidative or hydrolytically driven process, except rate constants, kinetic regimes and diffusivity/solubility will be different. Reagent molecules (oxygen or water) diffuse into the material and a chemical consumption rate then results in a reduction in local concentration with increasing depth. The key question is whether the observed profile could be obtained by a reasonable balance between permeation and reactive consumption in units of mols/g-cc. The possibility of an equilibrium aging condition that could yield this profile with moderate maximum conversion (edge aging level) was therefore probed with existing models normally used to describe oxidative degradation processes [30] [31].

Empirical modeling showed that the observed shape of the field aging profile would be consistent with a single sided equilibrium oxidative process where a kinetic regime of zero order applies for the rate dependence on local reagent concentration. This behavior results in a steeper profile than is more commonly observed [30] [31]. Model predictions matched the observed profile for the following parameters: depth of $2.3 \mathrm{~mm}$, external $\mathrm{O}_{2}$ partial pressure of $16 \mathrm{cmHg}$, material density of $1.3 \mathrm{~g} / \mathrm{cc}$, beta factor of 100 (equates to a kinetic regime of zero order for the governing reaction), consumption rate of $3.310^{-12} \mathrm{~mol} / \mathrm{g}-\mathrm{cc}$, and a permeability of $510^{-11} \mathrm{ccSTP} / \mathrm{cm}-\mathrm{s}-\mathrm{cmHg}$. Over 23 years the integrated edge oxidation for this model will be an aging state or conversion of $7.5 \%$ oxidation, which is a very reasonable level yet consistent with a substantial aging process ultimately leading to a considerable hardness evolution. In such a model the critical balance is given between permeability and consumption rate. As long as this ratio remains constant identical profiles will evolve, yet the edge conversion level will depend on the oxidation rate, and with much higher rates in these simulations unrealistic oxidation levels would apply.

A transition from an oxidative to a hydrolytically driven process is rea- 
sonably straightforward. Diffusion limited degradation models balance permeability and consumption in terms of absolute numbers of local reagent molecules: hence, a water permeability and consumption rate of similar magnitude will result in the same underlying conversion profile. Of course how such a process translates local chemistry to hardness is a separate issue. On this note our basic model assessment considers a linear relationship between localized chemistry and hardness. Other relationships may exist, yet we do not believe that they may prevent a reasonable diffusion limited degradation model from fitting the observed profile.

From a modeling perspective, all indications are that a diffusion limited hydrolytic degradation process when in an equilibrium state could certainly yield the observed field aging profile. Similarly, an aging process that yields a profile that slowly shifts inwards with time and results in some edge aging level saturation is certainly feasible. As long as a moderate water consumption rate is coupled with a low permeability constant, a suitable kinetic regime is dominant, and any water consumption chemistry will translate to hardening, the observed profile will evolve.

\section{Conclusion}

Based on test results for a naturally aged sample used for 23 years at sea a large ageing effect was observed when silica filled polychloroprene was immersed in sea water. After 23 years in service, modulus at $100 \%$ elongation increased by a factor of 4 , while both stress and strain at break were reduced by $70 \%$. Furthermore, degradation led to gradients across the sample thickness, similar to diffusion limited oxidation (DLO) phenomena when oxidation is involved.

Because polymer ageing is a slow process it is necessary to perform accelerated ageing testing in the laboratory. Using natural renewed sea water tanks at different temperatures up to $80^{\circ} \mathrm{C}$ polychloroprene samples were aged for more than 1.5 years. Accelerated ageing led to the same mechanical changes in the polymer as natural ageing, such as an increase in the stiffness and a decrease in ultimate tensile properties. Here again, heterogeneities in degradation levels arose across the sample thickness, due to competition between reaction and diffusion.

Extrapolation of polychloroprene behavior was performed by applying a linear Arrhenius extrapolation to predict mechanical behavior in service conditions and compare with results obtained for naturally aged samples in order 
to evaluate the applicability of such predictions. It appears that a linear Arrhenius extrapolation is not useful for modulus and stress at break because of the degradation profile across sample thickness, at least. Concerning strain at break, it seems that the good correlation with an Arrhenius extrapolation may be explained by the fact that elongation at break is governed by the weakest point in the sample, i.e. in this case the external surface of the sample which is directly in contact with water.

\section{Acknowledgements}

The authors would like to thank the Bretagne region for its financial support, all the contributors to the FEMEM project, especially V. Laguarrigue from TRELLEBORG Engineered Systems for providing the materials, P. Charrier for helpful discussions, abd E. Breton and M. Broudin for careful measurements. P. Davies from IFREMER is also acknowledged for his careful reading of this paper. Thanks are due to $\mathrm{M}$. Celina for valuable comments in support of this work.

\section{References}

[1] Observatoire Français des Techniques Avancées, Vieillissement et durabilité des matériaux, vol. 2 of Arago 28, OFTA, 2003.

[2] ISO 11346, Rubber, vulcanized or thermoplastic - Estimation of lifetime and maximum temperature of use, 1997 (revised 2004).

[3] P. Mott, C. Roland, Ageing of natural rubber in air and seawater, Rubber Chemistry and Technology 74 (2001) 79-88.

[4] M. Celina, K. Gillen, R. Assink, Accelerated aging and lifetime prediction: review of non-Arrhenius behaviour due to two competing processes, Polymer Degradation and Stability 90 (2005) 395-404.

[5] P. Davies, G. Evrard, Accelerated ageing of polyurethanes for marine applications, Polymer Degradation and Stability 92 (2007) 1455-1464.

[6] K. Gillen, M. Celina, R. Clough, J. Wise, Extrapolation of accelerated aging data : Arrhenius or erroneous?, Trends in Polymer Science 5 (1997) 250-257. 
[7] F. Gugumus, Effect of temperature on the lifetime of stabilized and unstabilized PP films, Polymer Degradation and Stability 63 (1999) 4152.

[8] E. Richaud, F. Farcas, B. Fayolle, L. Audouin, J. Verdu, Hydroperoxide build-up in the thermal oxidation of polypropylene A kinetic study, Polymer Degradation and Stability 92 (1) (2007) 118-124.

[9] X. Colin, L. Audouin, J. Verdu, M. Le Huy, Kinetic modelling of the thermal oxidation of polyisoprene elastomers. Part 2: Effect of sulfur vulcanization on mass changes and thickness distribution of oxidation products during thermal oxidation, Polymer Degradation and Stability 92 (5) (2007) 898-905.

[10] F. Delor and J. Lacoste and J. Lemaire and N. Barrois-Oudin and C. Cardinet, Photo- and thermal ageing of polychloroprene: effect of carbon black and crosslinking, Polymer Degradation and Stability 53 (1996) 361-369.

[11] M. Celina, J. Wise, D. Ottesen, K. Gillen, R. Clough, Oxidation profiles of thermally aged nitrile rubber, Polymer Degradation and Stability 60 (1998) 493-504.

[12] M. Celina, J. Wise, D. Ottesen, K. Gillen, R. Clough, Correlation of chemical and mechanical property changes during oxydative degradation of neoprene, Polymer Degradation and Stability 68 (2000) 171-184.

[13] G. Li, J. Koenig, FTIR imaging of oxidation of polyisoprene 2. The role of N-phenyl-N'-dimethyl-butyl-p-phenylenediamine antioxidant, Polymer Degradation and Stability 81 (3) (2003) 377-385.

[14] P. Le Gac, D. Choqueuse, M. Paris, G. Recher, C. Zimmer, D. Melot, Durability of polydicyclopentadiene under high temperature, high pressure and seawater (offshore oil production conditions), Polymer Degradation and Stability 98 (3) (2013) 809-817.

[15] J. Unsworth, Y. Ng, Effects of immersion in sea water on the properties of polymers used in special marine cable, in: Polymers in Marine Environments, London (Great-Britain), 73-76, 1985. 
[16] K. Ab-Malek, A. Stevenson, The effect of 42 years immersion in seawater on natural rubber, Journal of Materials Science 21 (1986) 147154 .

[17] J. Pegram, A. Andrady, Outdoor weathering of selected polymeric materials under marine exposure conditions, Polymer Degradation and Stability 26 (1989) 333-345.

[18] D. Oldfield, T. Symes, Long term natural ageing of silicone rubber, Polymer Testing 15 (1996) 115-128.

[19] M. Rutkowska, K. Krasowska, A. Heimowska, I. Steinka, H. Janik, Degradation of polyurethanes in sea water, Polymer Degradation and Stability 76 (2002) 233239.

[20] X. Leveque, Behaviour of various elastomers in a marine environment, in: Polymers in Marine Environments, London (Great-Britain), 111116, 1985.

[21] A. Stevenson, Rubber in offshore engineering, Adam Hilger Ltd, 1984.

[22] M. Bowditch, K. Stannard, Effects of water absorption on the properties of a filled elastomer, in: Polymers in Marine Environments, London (Great-Britain), 117-119, 1985.

[23] V. Le Saux, Fatigue et vieillissement des élastomères en environnements marin et thermique: de la caractérisation accélérée au calcul de structure, Ph.D. thesis, Université de Bretagne Occidentale, 2010.

[24] W. Oliver, G. Pharr, A new improved technique for determining hardness and elastic modulus using load and sensing indentation experiments, Journal of Materials Research 77 (1992) 1564-1582.

[25] K. Gillen, R. Clough, C. Quintana, Modulus profiling of polymers, Polymer Degradation and Stability 17 (1987) 31-47.

[26] P. Le Gac, V. Le Saux, M. Paris, Y. Marco, Ageing mechanism and mechanical degradation behaviour of polychloroprene rubber in a marine environment: comparison of accelerated ageing and long term exposure, Polymer Degradation and Stability 97 (2012) 288-296. 
[27] K. Gillen, R. Bernstein, D. Derzon, Evidence of non-Arrhenius behaviour from laboratory aging and 24-year field aging of polychloroprene rubber materials, Polymer Degradation and Stability 87 (2005) 57-67.

[28] D. Bauer, J. Baldwin, K. Ellwood, Correlation of Rubber Properties between Field Aged Tires and Laboratory Aged Tires, Rubber Chemistry and Technology 78 (5) (2005) $777-792$.

[29] J. Wise, K. Gillen, R. Clough, An ultrasensitive technique for testing the Arrhenius extrapolation assumption for thermally aged elastomers, Polymer Degradation and Stability 49 (3) (1995) 403 - 418.

[30] K. Gillen, R. Clough, Rigorous experimental confirmation of a theoretical model for diffusion-limited oxidation, Polymer 33 (1992) 4358-4365.

[31] J. Wise, K. Gillen, R. Clough, Quantitative model for the time development of diffusion-limited oxidation profiles, Polymer 38 (1997) 19291944. 